\title{
Color Image Restoration Using Iterative Mead's Filter
}

\author{
Ayad A. A1-Ani, Mohammed Sahib Mahdi Altaei and Sally Ali Abdul Ameer Alrubaie \\ Department of Computers, College of Science, Al-Nahrain University, Jadriyah, Baghdad-Iraq. \\ Corresponding author: sally.lally89@gmail.com
}

\begin{abstract}
Image restoration is reconstructing the true image starting from degraded (blurred and noisy) image version. This problem could be handled as blind or non-blind mode depending on whether functional knowledge or point spread function (PSF) knowledge is available. These knowledge are related to the type and parameters of the additive noise such as; distribution, mean, and variance. Accordingly, the present work aims to restore the original image using adaptive Mead's algorithm applied on degraded version. The proposed method is individually applied on the three color components; red, green, and blue (RGB) of the image. The results are quantitatively and qualitatively compared to the original one using two quality measures, they are: mean squared error (MSE) and cross correlation coefficient (CCC). Results showed valued performance of the proposed method when restoring the degraded images. Quality measures proved that the blue component was reconstructed better than the red, and the red was reconstructed better than the green component. Frequent tests showed the matching score between the reconstructed image and the original one was about $97 \%$, which ensure the validation of the proposed method and correct path of computations. [DOI: 10.22401/ANJS.21.4.12]
\end{abstract}

Keywords: Image restoration, Mead's filter, blurring, noising.

\section{Introduction}

Image restoration is a method of improving the looks of the digital image. The primary goal of the image restoration algorithms is to undo the blurring function and the noise that degraded image by de-convolving the blurring function and minimize the noise of the degraded image to produce an estimate image, which is approach to the original image [1] [2]. The field of image restoration, image deblurring or image de-convolution concerns with an estimation of the uncorrupted image from degraded one. The restoration technique of the image is classified into degradation phase and restoration phase [3].

The degradation phase is concerned with the real image that degraded by the blurring and the extra noise. The resultant image of this part is called the degraded image. Whereas, the restoration phase is concerned with using many filters up on degraded image and estimating a picture for the original image to be produced as an output.Whereas, the image restoration methods are classified to blind class and non-blind class [4] [5]. Blind method of restoration which the blurring factor is unknown. and the Primary knowledge of $h(x, y)$ not needed but the blurring factor estimation necessary to use to de-blur the image. The non-blind method of restoration which the blurring factor is known and the prior knowledge of $h(x, y)$ required. Removing the blurs from the degraded images are conditioned on the blurring function knowledge [6] [7]. The degradation model is result from the convolution between the blurring function and real image with the effect of the additive noise function. The model which presents the degradation process in spatial domain is [8]:

$$
g(x, y=h(x, y) \circledast \mathrm{f}(\mathrm{x}, \mathrm{y})+\mathrm{n}(\mathrm{x}, \mathrm{y})
$$

Where $g(x, y)$ presents the degraded version of image $f(x, y)$ presents the original version of the image, $n(\mathrm{x}, \mathrm{y})$ presents the additive noise function and $\mathrm{h}(\mathrm{x}, \mathrm{y})$ presents the blaring function, the model which presents the degradation process in the frequency domain is [8][9]:

$$
\mathrm{G}(\mathrm{u}, \mathrm{v})=\mathrm{H}(\mathrm{u}, \mathrm{v}) \cdot \mathrm{F}(\mathrm{u}, \mathrm{v})+\mathrm{N}(\mathrm{u}, \mathrm{v})
$$

where, $(u, v)$ represent a spatial frequency coordinates, $\mathrm{G}(\mathrm{u}, \mathrm{v}), \mathrm{H}(\mathrm{u}, \mathrm{v}), \mathrm{F}(\mathrm{u}, \mathrm{v})$ and $\mathrm{N}(\mathrm{u}, \mathrm{v})$ is the Fourier transform for $g(x, y), h(x, y), f(x, y), n(x, y)$, respectively. 
The convolution in the spatial domain is equal the multiplication operation in the frequency domain or Fourier domain, in this restoration model, the real image and the noise are combined linearly, so that the problem of restoring the real image from the distorted image is defined as a linear image restoration problem.

The algebraic approach is the concept of finding an estimate of original picture, which minimizes a predefined criterion of performance. Because of simplicity, the approach attentions on least-squares criterion function. The well-known restoration methods are due to considering one of the process either an unconstrained or a constrained process to least-square restoration problem [8]. According to the effect of blurring function (PSF), whether it is space invariant or space variant image restoration can be classified into linear and non- linear restoration. The linear restoration is associated with space invariant PSF, while the non-linear restoration is associated with space variant PSF. The linear restoration techniques can be categorized for two techniques: unconstrained linear restoration, and constrained linear restoration. Also, the constrained techniques are classified into direct solution (also known as, linear, non-iterative, or one shot solution), and indirect solution that known as iterative solution [10]

\section{Mead's Filter Reconstruction}

Image reconstruction using Mead's filter uses the iterative non- blind Mead's algorithm, which is a linear and constrained restoration technique. Mead's filter related to the least square method that obtains the result of restoration iteratively non in one shot solution. The Mead's filter adaptive to constrained least square method that find the optimal estimate for $\hat{f}$ of the un-known target function $\mathrm{f}$ and generally formulated as constrained optimization problem to find $\min \|C \hat{f}\|^{2}$ that seems to be acceptable at the following condition:

$$
\|g-H \hat{f}\|^{2}<\epsilon^{2}
$$

where $\mathrm{C}$ is suitable weighting matrix, $\mathrm{C}$ could be a Laplacian operator or signal to noise ratio. The constrained problem can be transformed to unconstrained problem by using Lagrangian multiplier method so the estimate for $\hat{f}$ found by reducing the error function with respect to $\hat{f}$ as follows:

$$
E_{1}(\hat{f})=\|g-H \hat{f}\|^{2}+\alpha\|C \hat{f}\|^{2}
$$

The solution is usually found by $\frac{\partial E_{1}}{\partial \hat{f}}=0$ one shot solution since no iteration involved

$$
\hat{f}=\left[H^{\sim} H+\alpha C^{\sim} C\right]^{-1} H^{\sim} g
$$

where, $\alpha$ is regularizing parameter that controls the tradeoff between the amount of restoration and amount of noise. The solution of equation (5) is found iteratively by[11][12]

$$
\hat{\mathrm{f}}_{\mathrm{k}+1}=\hat{\mathrm{f}}_{\mathrm{k}}+\beta\left[\mathrm{H}^{\sim} \mathrm{g}-\left(\mathrm{H}^{\sim} \mathrm{H}+\alpha \mathrm{C}^{\sim} \mathrm{C}\right) \hat{\mathrm{f}}_{\mathrm{k}} \ldots\right.
$$

Where $\beta$ control the convergence of the iterations. The damped least square (DLS) restoration method represented as constrained optimization problem of $\min _{\Delta \hat{f}} \Delta \hat{f}^{\sim} C \Delta \hat{f}$ that constracted at the following condition:

$$
\mathrm{S}, \mathrm{t}\|g-H \hat{f}\|<\epsilon
$$

Where $\Delta \hat{f}$ is correction vector or optimization step and $\mathrm{C}$ diagonal weighting matrix. The DLS even represented as unconstrained problem of

$\min _{\Delta \hat{f}}\|g-H\|+\alpha^{2} \Delta \hat{f}^{\sim} C \Delta \hat{f}$, where, $\quad \alpha$ is regularizing parameter. The restoration problem is solved iteratively by Mead's recurrence method by the following equation [11]:

$$
\begin{aligned}
& \hat{f}_{k+1}=\hat{f}_{k}+\left[H^{\sim} H+\alpha C\right]^{-1} H^{\sim}\left(g-H^{\sim} \hat{f}_{k}\right) \\
& \hat{f}_{k+1}=\hat{f}_{k}+w\left(g-H^{\sim} \hat{f}_{k}\right)
\end{aligned}
$$

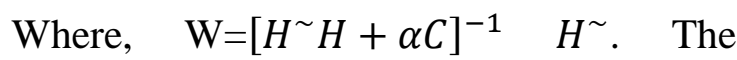
following subsections explain the theoretical concepts of both the basic iterative algorithm and the way of the de-blurring procedure:

\section{Basic Iterative Algorithm}

The basic iterative algorithm depends on assuming the restored image is empty, and then it take few details at each iteration, this is can be expressed by the following two relations: 


$$
\begin{aligned}
& \hat{f}=0 \ldots \ldots \ldots \ldots \ldots \ldots \\
& \hat{f}_{k+1}=\hat{f}_{k}+\beta\left(g-H \hat{f}_{k}\right)
\end{aligned}
$$

Where $\mathrm{k}$ is number of iteration, $\hat{f}_{k+1}$ is restored image after $\mathrm{k}+1$ iterations, $\hat{f}_{k}$ is restored image after $\mathrm{k}$ iterations, $\beta$ controls the convergence of the iterations. The easiest iterative restoration technique have a long history, it belongs back at least to the VanCittert's work in the 1930's, and may in fact have even older antecedents [13]. VanCittert's case was specially invariant and used $\beta=1$ [14], Jansson made a modification on VanCittert's work by replace the gain $\beta$ by relaxiation parameter that depend on signal[15], even kawata used iterative restoration method with fixed and vary parameter $\beta[16][17]$. Iterative restoration algorithms need a first estimate to start their iterations to be given as follows [1]:

- Determine the recorded image, which is the first estimate that refers to the degraded image.

- Determine the mean of the recorded image, Kaufman (1987) guesses a good approach shot if no other suitable guess as a first estimate can be obtained by use the mean of degraded image as uniform first estimate [18].

- The real target ideally, the restoration algorithms should produce the real target. Utilizing the original object as a first estimate enables us to test whether this is the solution found by these algorithms under realistic circumstances.

- The result of Tikhonov-Miller restoration, in which the iterative algorithms based on the Tikhonov functional should converge more quickly on their solutions if a first estimate close to the final solution which is provided [13].

\section{De-blurring Procedure}

The basic form of iterative restoration filters is the one that iteratively approaches the solution of the inverse filter, and is given by the following spatial domain iteration:

$$
\begin{aligned}
& \hat{f}_{k+1}(\mathrm{x}, \mathrm{y})=\hat{f}_{k}(x, y)+\beta(g(x, y)- \\
& \left.H(x, y) \hat{f}_{k}(x, y)\right)
\end{aligned}
$$

where, $\hat{f}_{k+1}(\mathrm{x}, \mathrm{y})$ is the restoration result after $i$ iterations. Usually in the first iteration $\hat{f}_{k}(x, y)$ is chosen to be identical to zero or identical to $g(x, y)$. The 25th iteration has been independently discovered many times, and is referred to as the van Cittert, Bially, or Land weber iteration. As can be given in eq. (12), during the iterations the blurred version of the current restoration result $\hat{f}_{k}(x, y)$ is compared to the image $g(x, y)$. The difference between the two is scaled and added to the current restoration result to give the next restoration result. Deblurring is equivalent to applying the VanCittert procedure to the identity given by the following relation [13]:

$$
\hat{f}=\hat{f}+\beta H^{t}(g-H \hat{f})
$$

Forming the iteration gives the following relation [13]:

$$
\hat{f}_{k+1}=\hat{f}_{k}+\beta H^{t}\left(g-H \hat{f}_{k}\right)
$$

An alternative way to derive Eq. (14) is from the view point of minimizing the norm of the residual $(g-H \hat{f})$ because for a good restoration result the blurred estimate $H \hat{f}$ should be approximately equal to the observed image $g$ [19]

\section{Proposed Image Reconstruction Method}

The generic structure of the proposed image restoration method is shown in Fig.(1). In addition to the preprocessing stage, the proposed method is designed to be consisted of two phases: degradation and restoration. The degradation is an image preparation phase, which is responsible on impact the quality of test image by the degradation process that includes blurring and noising the test image. Whereas the restoration is a post processing phase that responsible on restore the quality of the image by using the filtering technique, which is carried out by the spatial domain using Mead's filter. More explanations related to the degradation and restoration phases are given in the Fig.(1)

\section{Preprocessing Stage}

The test image $(f)$ is loaded in three color components: red $(R)$, green $(G)$, and blue $(B)$, these components are contributed together to introduce the material colored and grey images 
that used are in following processing. The preparing is a primary stage aims at preparing the test image for highlighting the image cues. Image color transformation is an important stage for concentrating the information carried in three bands to be dense in one grey band. Thus, the three estimated bands $R, G$, and $B$ are converted into single band according to YIQ color transformation system [20]. The $Y$ represents the intensity band, whereas both $I$ and $Q$ represent the chrominance bands. Just the $Y$ band is useful in the present work, which can be denoted as $f_{T}(x, y)$ and computed according to the following relation:

$f_{T}(x, y)=0.299 \times R(x, y)+0.587 \times$

$G(x, y)+0.114 \times B(x, y)$

\section{Degradation Phase}

The degradation phase is composed of two stages: blurring stage and noising stage. Both mentioned stages are begin with producing a Gaussian distribution as two dimensional function, and then including this function within the image. The generation of Gaussian function depends on using the Gaussian distribution at a specific useful value of standard deviation $(\sigma)$. Such process requires determining the kernel size $\left(S_{k}\right)$ that represents the affected area of the image segment when applying the Gaussian function for blurring the image. The blurring stage is responsible on smoothing the test image. Blurring leads to embed some fine details in the image by convolving the Gaussian function with the original image. The noising stage is implemented in the spatial domain which requires first to estimate the noise function of a specific SNR. Noisy image is resulted by combining the generated noise function with the original image. The combination of additive white noise to the blurred colored image includes the process of adding three random numbers are chosen from the Gaussian distribution to the three color channels of the blurred colored image, in which each random number of a specific channel is different from that of other channels. The use of the blurred image $(b)$ instead of the original one with the effect of additive noise is produce the degraded image $(d)$.

\section{Restoration Phase}

The restoration phase includes the restoration filter that suggested to be used. The meads filter is an iterative one that goes to correct the restored value of the image pixels at each time the iteration counter increased. The spatial domain based image restoration includes creation of circulant matrix $(c)$ that needed to be used in the application of the meads filter. The circulant matrix and circulant shifting are used to filter the degraded image and producing the restored image (d) iteratively by eq.(14).

\section{Restoration Measurements}

The image quality measurements that are used to evaluate the proposed restoration method are applied on the resulted restored image comparing with the original image. The adopted quality measures are: signal to noise ratio (SNR), and mean squared error (MSE). Given by the following eq(s):

$\operatorname{MSE}=\frac{1}{M N} \sum_{x=1}^{N} \sum_{y=1}^{M}[f(x, y)-g(x, y)]^{2}$

Where, $f(x, y)$ is the original picture or the real picture or the degradation-free image with a very good quality, $\mathrm{g}(\mathrm{x}, \mathrm{y})$ is the degraded image of $f(x, y)$, whose quality evaluated. The higher MSE indicates more difference between the real and processed image [21]. Signal to noise ratio (SNR) is a mathematical measure for evaluating image quality based on the pixel difference between two pictures, it defines the relationship between the original image and estimated image. This ratio shows how strong the noise distorted the real image. The signalto-noise-ratio of the restored image is similarly defined as:

$S N R_{f^{\wedge}}=10 \log _{10}\left[\frac{\operatorname{var} f(x, y)}{\operatorname{var} f^{\wedge}(x, y)-f(x, y)}\right]$

The use of such quality measurements is due to their high capability of describing the filtering performance that leads to gives restore acceptable quality image in comparison with the reference one. This can evaluate the validation test of the proposed method [9]. 


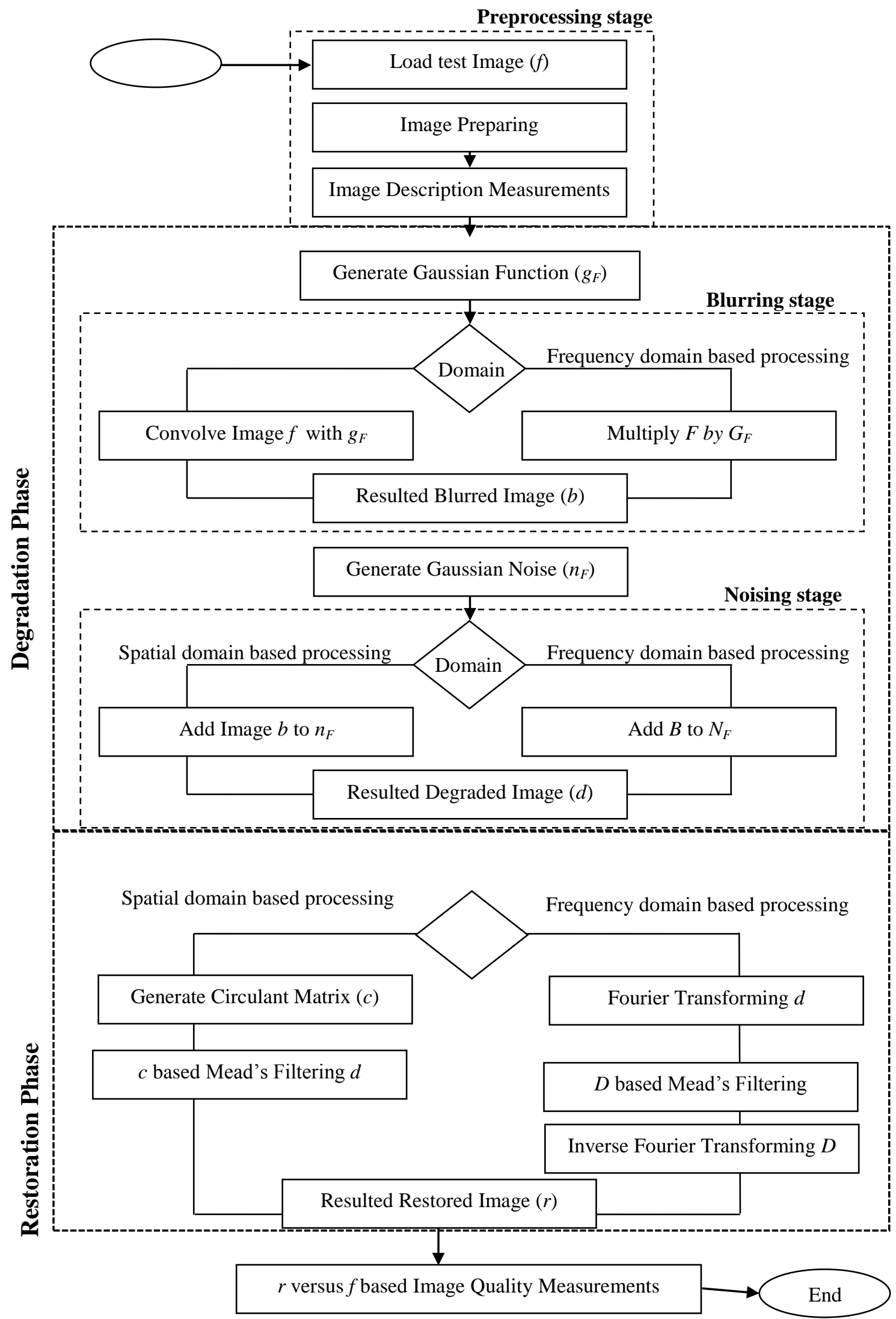

Fig.(1): Generic structure of proposed image restoration method. 


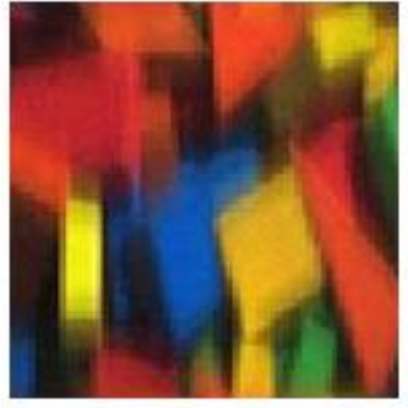

(a) $\sigma=1, S N R=30$

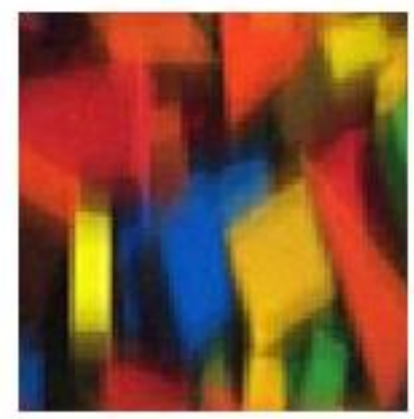

(d) $\sigma=2, S N R=30$

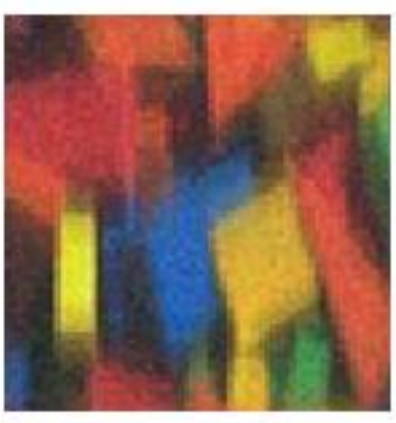

(b) $\sigma=1, S N R=20$

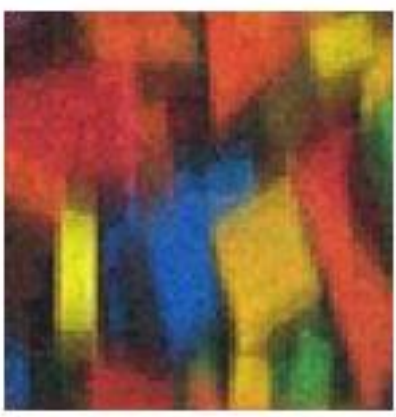

(e) $\sigma=2, S N R=20$

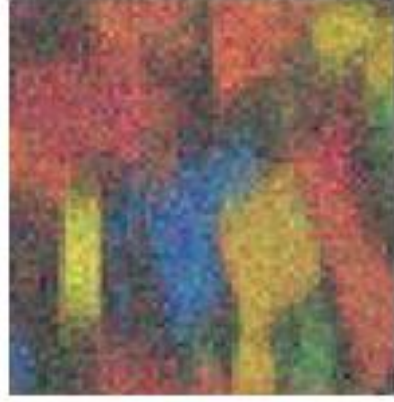

(c) $\sigma=1, S N R=10$

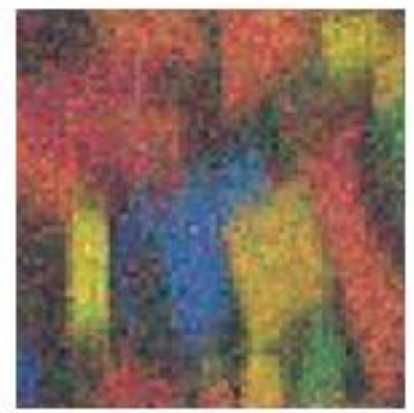

(f) $\sigma=2, S N R=10$

Fig.(1): (a-c) Degraded image with PSF of $\sigma=1$ (d-f) Degraded image with PSF of $\sigma=2$ and restorted with noise $S N R=30,20$ and 10 respectively.

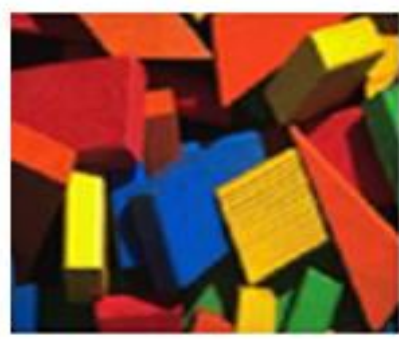

(a) Original

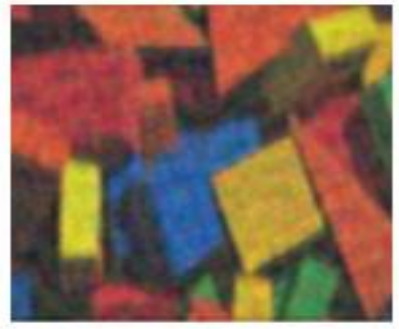

(d) $I=10, \mathrm{MSE}=381$

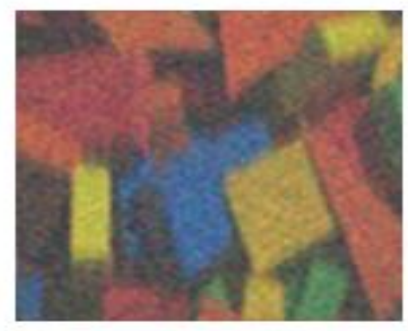

(b) $\mathrm{MSE}=1460$

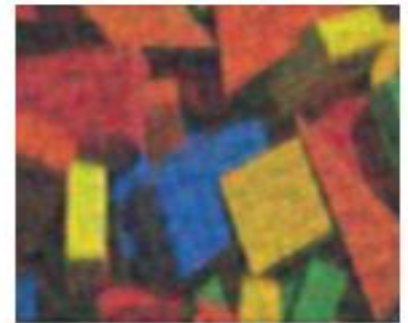

(e) $I=15, \mathrm{MSE}=374$

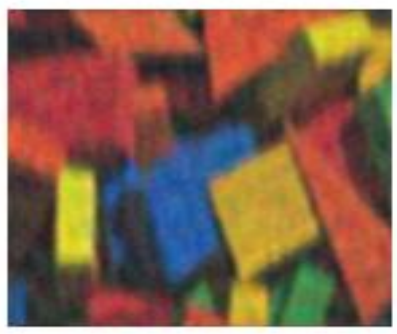

(c) $I=1, \mathrm{MSE}=420$

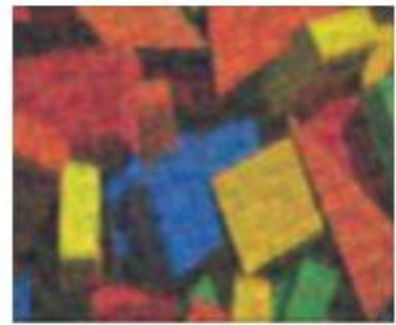

(f) $I=20, \mathrm{MSE}=370$

Fig.(2): (a) Original image (b) Degraded image with PSF of $\sigma=1$ and noise with $S N R=10 \mathrm{~dB}(c-f)$ Restored image after:1,10,15 and 20 iteration. 


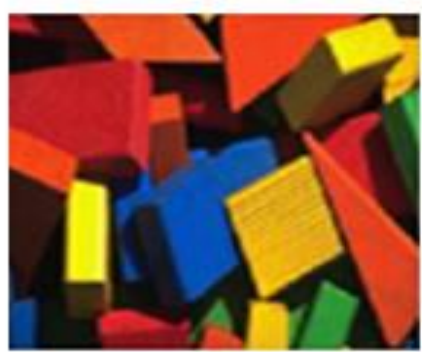

(a) Original

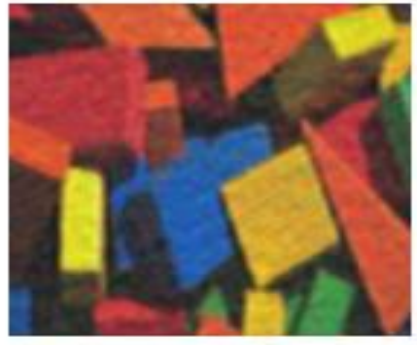

(d) $I=10, \mathrm{MSE}=158$

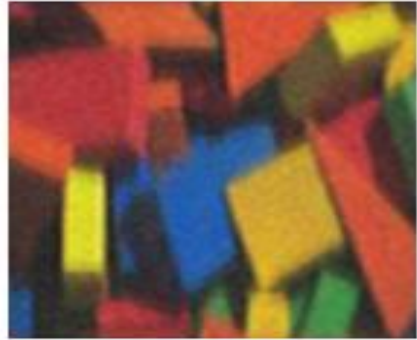

(b) $\mathrm{MSE}=341.5$

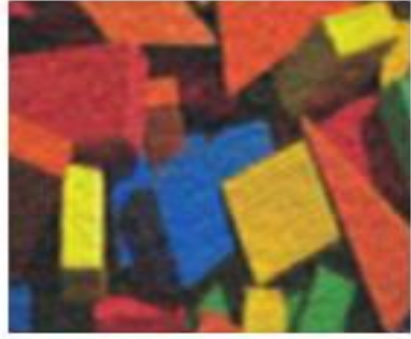

(e) $I=15, \mathrm{MSE}=156$

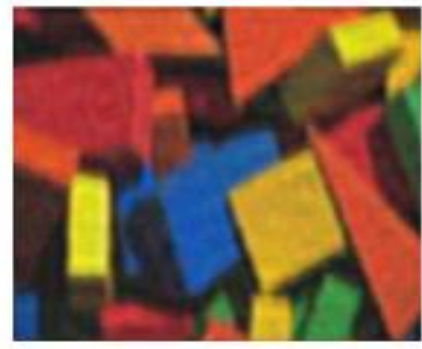

(c) $I=1, \mathrm{MSE}=193$

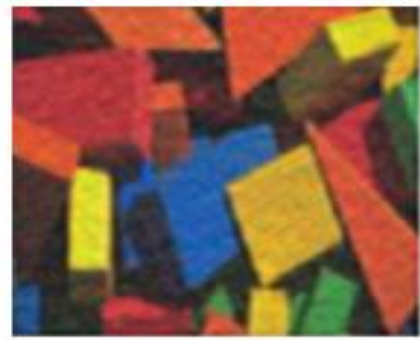

(f) $I=20, \mathrm{MSE}=155$

Fig.(3): (a) Original image (b) Degraded image with PSF of $\sigma=1$ and noise with $S N R=20 \mathrm{~dB}(c-f)$ Restored image after:1,10,15 and 20 iteration.

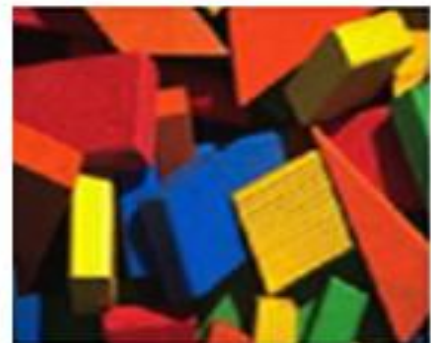

(a) Original

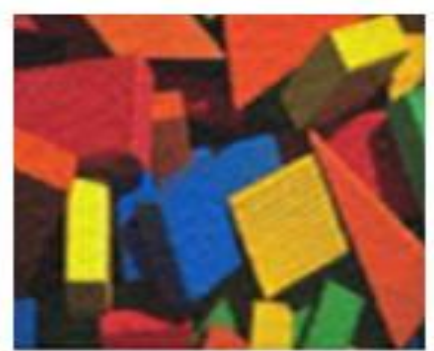

(d) $I=10, \mathrm{MSE}=55$

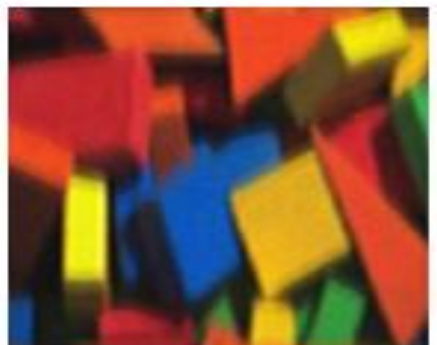

(b) $\mathrm{MSE}=228.7$

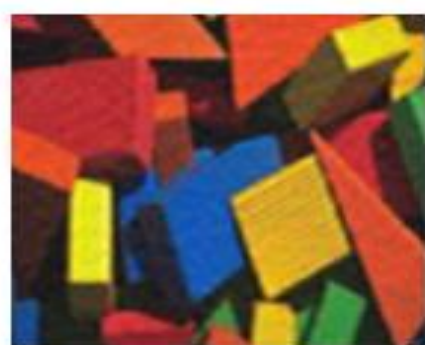

(e) $I=15, \mathrm{MSE}=54.5$

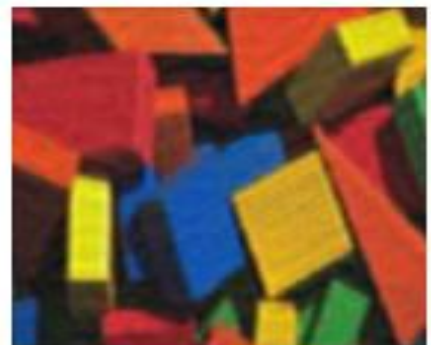

(c) $I=1, \mathrm{MSE}=92$

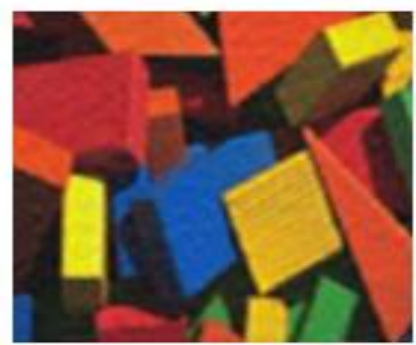

(f) $I=20, \mathrm{MSE}=53$

Fig.(4):(a) Original image (b)Degraded image with PSF of $\sigma=1$ and noise with $S N R=30 \mathrm{~dB}(\mathrm{c}-\mathrm{f})$ Restored image after:1,10,15 and 20 iteration. 


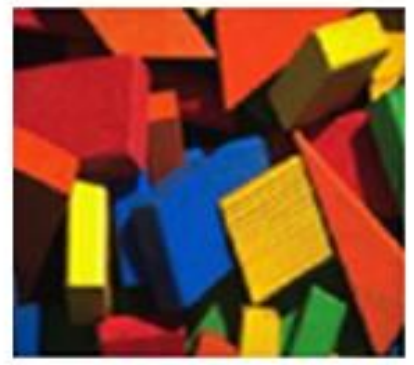

(a) Original

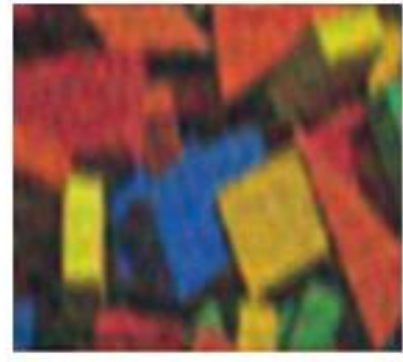

(d) $I=10, \mathrm{MSE}=410$

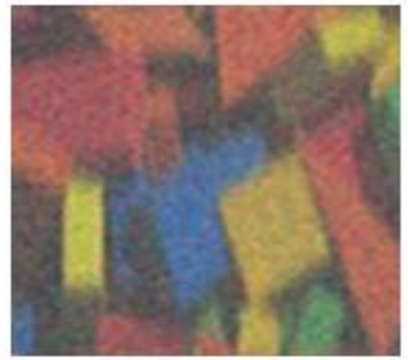

(b) MSE $=1553$

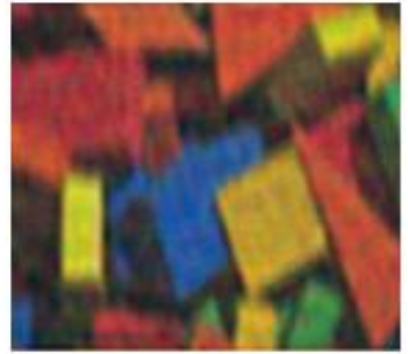

(e) $I=15, \mathrm{MSE}=406$

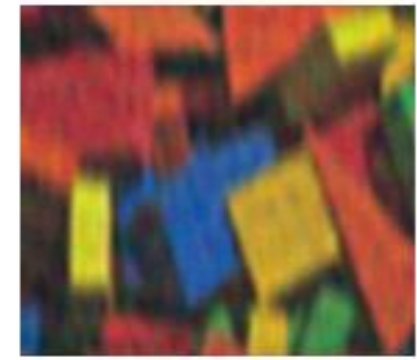

(c) $I=1, \mathrm{MSE}=434$

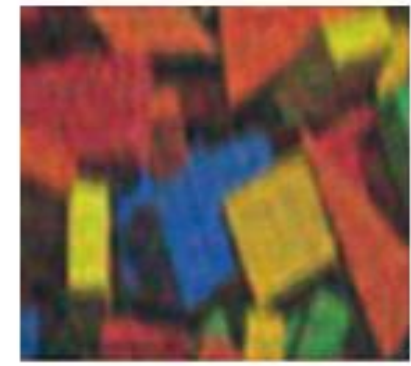

(f) $I=20, M S E=405$

Fig.(5): (a) Original image (b) Degraded image with PSF of $\sigma=2$ and noise with $S N R=10 \mathrm{~dB}(c-f)$ Restored image after:1,10,15 and 20 iteration.

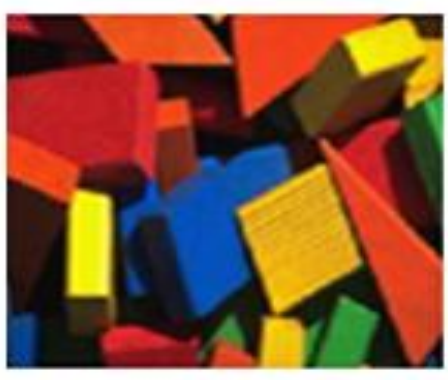

(a) Original

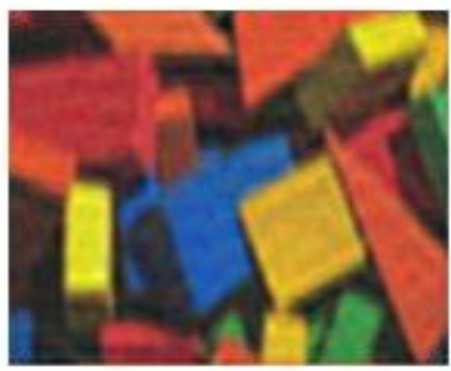

(d) $I=10, \mathrm{MSE}=247$

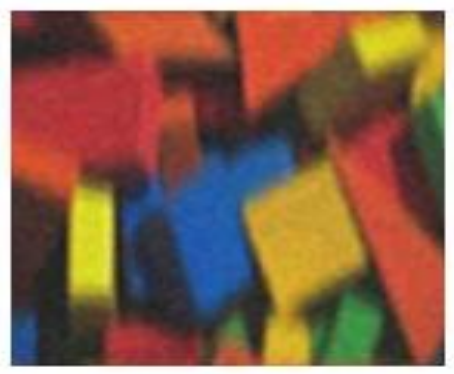

(b) $\mathrm{MSE}=451$

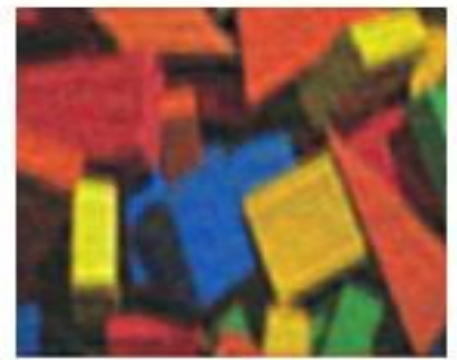

(e) $I=15, \mathrm{MSE}=244$

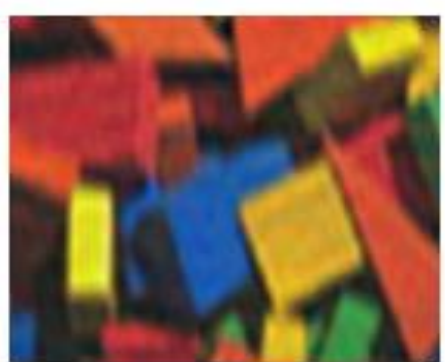

(c) $I=1, \mathrm{MSE}=278$

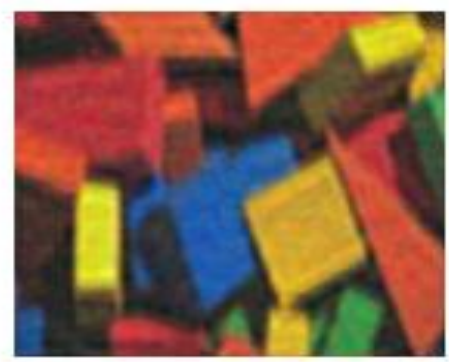

(f) $I=20, \mathrm{MSE}=243$

Fig.(6): (a) Original image (b) Degraded image with PSF of $\sigma=2$ and noise with $S N R=20 \mathrm{~dB}(\mathrm{c}-f)$ Restored image after:1,10,15 and 20 iteration. 


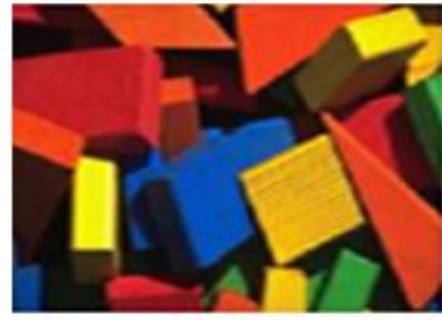

(a) Original

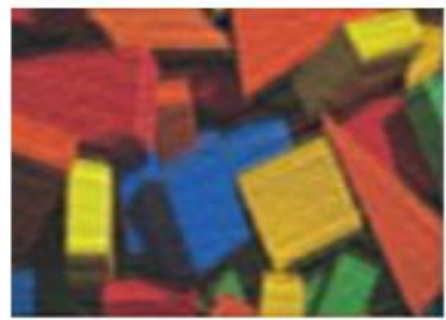

(d) $I=10, \mathrm{MSE}=171$

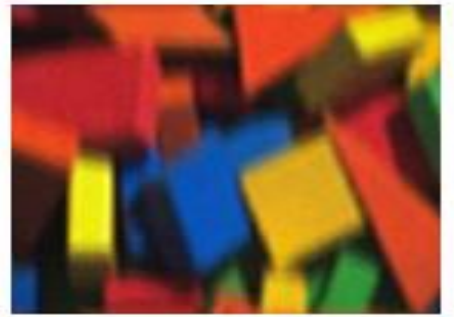

(b) $\mathrm{MSE}=340$

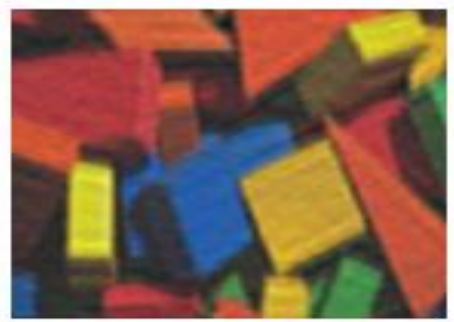

(e) $I=15, \mathrm{MSE}=168$

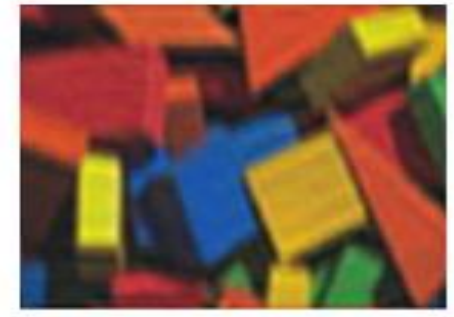

(c) $I=1, \mathrm{MSE}=211$

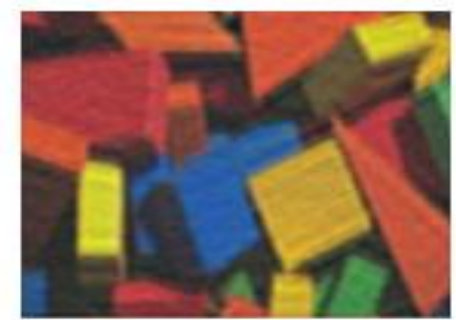

(f) $I=20, \mathrm{MSE}=166$

Fig.(7): (a) Original image (b) Degraded image with PSF of $\sigma=2$ and noise with $S N R=30 \mathrm{~dB}(c-f)$ Restored image after:1,10,15 and 20 iteration.

It is shown that the quality of the restored image improves with increasing the number of iterations. In such case, some amounts of the blur and noise are eliminated and the image is reconstructed time by time. Therefore, the best quality image is that resulted when the number of the iteration is considered to be at maximum enough value. Actually, the effective performance of the used Meads filter make the image to be in acceptable form when the iteration number is 20. More iteration does not offer noticeable improvements in the restored image. It is shown that the quality of the restored image is increased with less value of $\sigma$ and greater value of $S N R$, this interprets that the best restored image is yield when $\sigma=1$ and $S N R=30$. The least $\sigma$ refers to less smoothing occur in the image and less embedded cues are happen, in which the edges appear with small amount of smoothing is proportional to the breadth of the effected blurring function. Also, the greater value of SNR that shows better quality of restored image is due to the increase of the useful signal comparing with the assumed noise, where the useful signal is the information of the image cues. It is noticeable that the restoration results are in qualitative measurement appearing closer to the original image with decreasing $\sigma$ and increasing $S N R$, best result is when $\sigma=1$ and $S N R=30$. Also, it is found that the number of iteration affects the restoration results, more accurate results are obtained at more iteration number.

\section{Results Evaluation}

The results of the proposed image restoration method by Meads filter in the spatial domain can be evaluated by noting the comparison results between the restored image obtained by Meads filter with the original image. The quality measures of interest are the MSE and CCC

The MSE indicates the amount of the divergence between the restored image and the reference one, while the $\mathrm{CCC}$ indicates the amount of correlation between the restored image pixels with those of the reference image at same spatial locations. Fig.(8 and 9) show the MSE and CCC behaviors measured for the restored considered case in comparison with the original test image. 


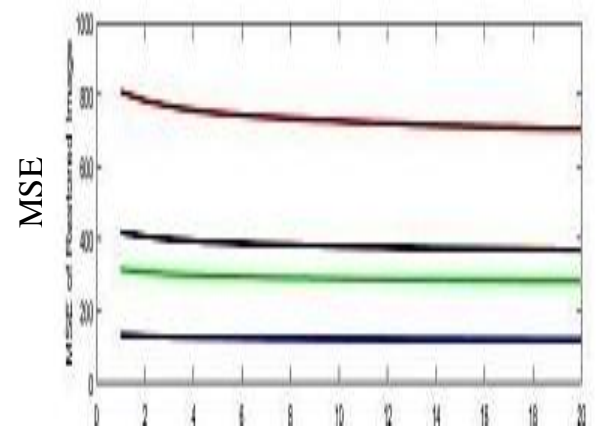

$\mathrm{Nu}$. of iterations

(a) $\sigma_{\mathrm{e}}=1, S N R=10$

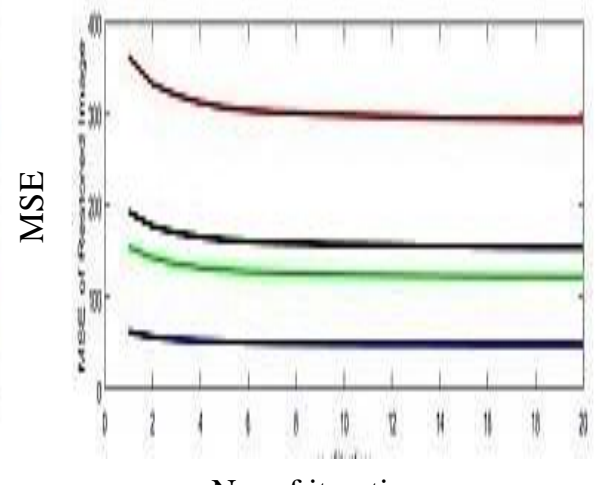

$\mathrm{Nu}$. of iterations

(b) $\sigma_{\mathrm{e}}=1, S N R=20$

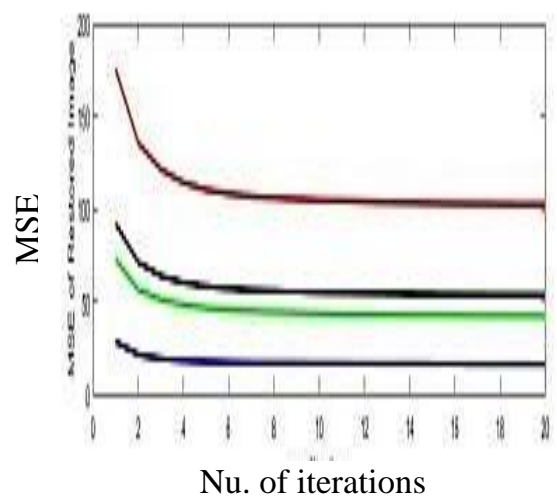

(c) $\sigma_{\mathrm{e}}=1, S N R=30$

Fig.(8): Resulted MSE between the restored image and original at different cases of $\sigma$ and $S N R$.

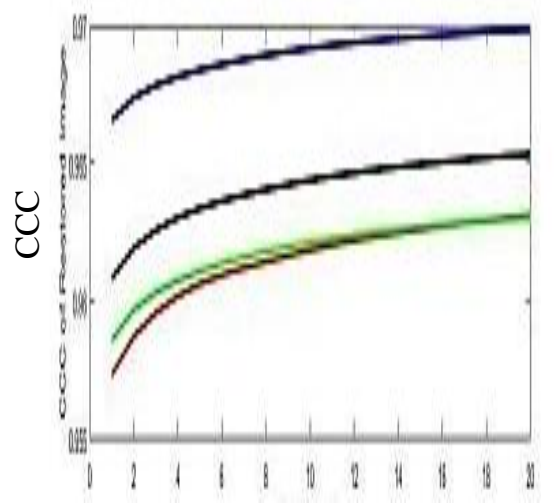

$\mathrm{Nu}$. of iterations

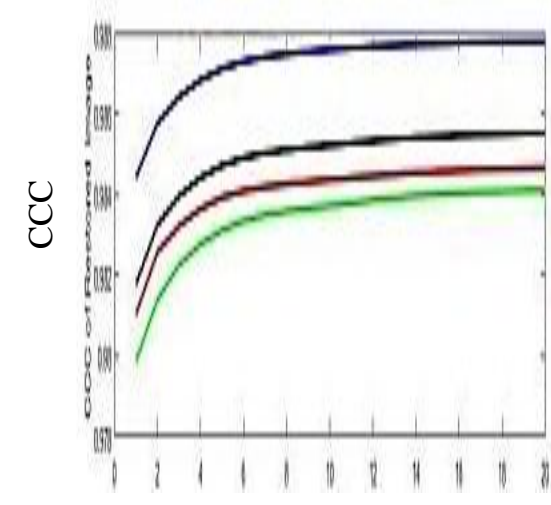

Nu. of iterations

(b) $\sigma_{\mathrm{e}}=1, S N R=20$

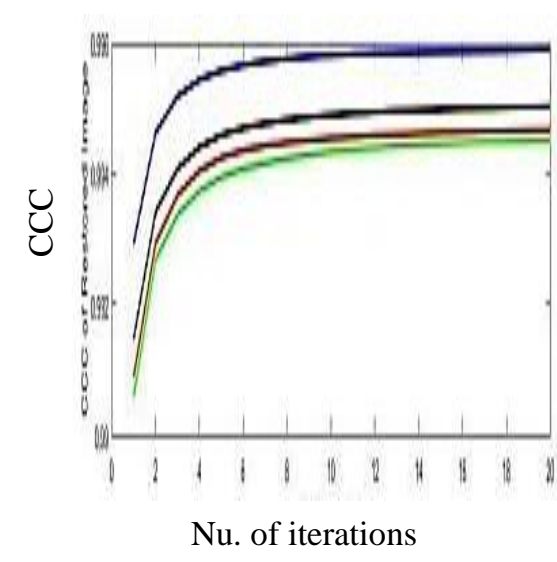

(c) $\sigma_{\mathrm{e}}=1, S N R=30$

Fig.(9): Resulted CCC between the restored image and original at different cases of $\sigma$ and SNR.

It is shown that the MSE and CCC are computed for the three color components of the restored color image for cases at which the estimated $\sigma$ is: $\sigma_{e}=1$ and the estimated SNR is: $S N R_{e}=10,20$, and 30. The case of $\sigma_{e}=1$ and $\mathrm{SNR}=10$ shows very little MSE and greater CCC. The least value of MSE is associated with greatest value of CCC at the case when $\sigma_{e}=1$ and $S N R_{e}=30$. In spite of the acceptable results of both MSE and CCC of test used color image, these results indicate that there is different performance may occur by the proposed filter at each color band of the origin image according to the amount of information and their distribution in that band. Generally, the average value of the MSE is about $3 \%$ for the restored color image, which indicates that the converging percent was $97 \%$ when considering the comparison between the restored image with the original image.

\section{Conclusions}

Results of using the iterative Mead's filter for restoring degraded images enable to conclude that the adopted technique is an efficient method to restore the degraded colored images with acceptable performance. The restoration performed well when using the blue component, the red component was located in the second place, and then the green component. Also, the MSE decreases with decreasing the blurring amount and also increasing the noise quantity. MSE decreases with increasing the number of iterations till reaching a specific number; where there is no restoration may be noticed. The first iteration had the better restoration than other iteration The behavior of the CCC was inversely behaved to the MSE with the blurring and noising that impact the original image. Quality measures proved that the blue component was reconstructed better than the red, and the red 
was reconstructed better than the green component and degradation on red component was mor than green and red so that the restoration curve decreasing more than other band because the red color amount more than other color on blocks image.

\section{References}

[1] Reginald L. Lagendijk, Jan Biemond. "Iterative Identification and Restoration of Images", Kulwer Academic, Boston, 1991.

[2] Aziz Makandar and ELS. "Computation Pre-Processing Techniques for Image Restoration". International Journal of Computer Applications,113(4), 11-17, 2015.

[3] Er. Priya Tiwari, Dr. Naveen Dhillon and Er. Kuldeep Sharma. "Analysis Of Image Restoration Techniques For Developing Better Restoration Method" International Journal for Science and Emerging Technologies with Latest Trends.19(1),1014, 2014.

[4] Tsung-Ying Sun, Sin-Jhe Ciou, ChanCheng Liu, Chih-Li Huo, "Out-of-focus blur estimation for blind image deconvolution Using particle swarm optimization" IEEE, Systems, Manand Cybernetics, 1627-1632, 2009.

[5] A. KAUR, V. Chopra, "A comparative study and analysis of image restoration techniques using different image formats" international journal for science and emerging technologies with latest trends 2(1):7-14., 2012.

[6] Dong-Dong Cao, Ping Guo, "Blind image restoration based on wavelet analysis," IEEE, Machine Learning and Cybernetics, 4977 - 49822005.

[7]R. C. Gonzalez and R. E. Woods, "Digital Image Processing Second Edition", Prentice-Hall India, 2007.

[8] Gonzalez R.C. , Wintz P., "Digital Image Processing", Addison-Wesley, Canada, 1987.

[9] Dejee Singh, Mr R. K. Sahu , "A survey of various image deblurring techniques", IJARCCE vol. 2, issue 12 december 2013.

[10] Mohammed K. Kadhom. "Image Restoration Using Adaptive Nonlinear Techniques". Msc. thesis. Department of Physics, Collage of Science, Al-Nahrain University. 2008.
[11] olli seger, "Model Building and Restoration with application in confoc'al Microscopy", department of electrical engineering, Linkoping university, Linkoping, Sweden. 1993.

[12] D.P.Lun, T.C.Chan, T.C.Hsung, D. D. Feng, and Y.H.Chan; "Efficient blind restor-ation using discrete periodic random transform"; IEEE Trans. Image Processing 13 188-200, 2004

[13] G. van Kempen and L. van Vliet, "The influence of the regularization parameter and the first estimate on the performance of Tikhonov regularized nonlinear image restoration algorithms," Journal of Microscopy, 198 (1),63-75, 2000.

[14] P.H Van Cittert, "Zum einfluss der spaltbreite auf die Intensitätsverteilung in Spektrallinien". II, Z. Physik, 69(5-6), 298308, 1930.

[15] P. A. Jansson, R. H. Hunt, and E. K. Plyler, "Resolution enhancement of spectra". J. Opt. Soc. Am. 60:596-599, 1970.

[16] S. Kawata, Y. Ichioka, and T. Suzuki, "Application of man-machine interactive image processing system to iterative image restoration", Proc. 4th Int. Conf. on Pattern Recognition, 525-529, Kyoto, 1978

[17] S. Kawata, Y. Ichioka, July. "Iterative image restoration for linearly degraded images. II. Reblurring procedure", J. Opt. Soc. Am., 70, 768-772, 1980.

[18] Angwin, D., Kaufman, H): "Adaptive restoration of color images. Proceedings of the $26^{\text {th }}$ Conference on Decision and Control, Los Angeles, CA, 1987.

[19] Aswathi V M and James Mathew. "A Review on Image Restoration in Medical Images". An international journal of advanced computer technology 4(4), 15881591. 2015.

[20] Gonzalez R.C, Wintz P, "Digital Image Processing", second Edition. AddisonWesley, 2002.

[21] J. Bartrina-Rapesta, F. Auli-Llinas, J. Serra-Sagrista, and J. Monteagudo-Pereira, "JPEG2000 Arbitrary ROI coding through ratedistortion optimizationtechniques," in Data Compression Conference, 25-27, 292 $-301.2008$. 